\title{
Multirresistencia, resistencia extendida y panresistencia a antibacterianos en el norte de México
}

\author{
Multidrug, extended and pan-resistance to antimicrobials at the North of México
}

Luis A. Camacho-Silvas 1,2, Jorge H. Portillo-Gallo2,3, Antonio E. Rivera-Cisneros', Jorge M. Sánchez-González ${ }^{5}$, Rafael Franco-Santillán 6 , Jorge Duque-Rodríguez ${ }^{1+}$, Gerardo Velo-Méndez y Cecilia Ishida-Gutiérrez ${ }^{1 *}$

${ }^{1}$ Laboratorio de Farmacoepidemiología, Facultad de Medicina y Ciencias Biomédicas, Universidad Autónoma de Chihuahua; '2Laboratorio Clínico, Hospital Star Médica Chihuahua; ${ }^{3}$ Deparrtamento Patología Clínica y Molecular, LIACSA Laboratorios Chihuahua; ${ }^{4}$ Departamento Medicina del Deporte, Universidad de Fútbol y Deporte, León, Guanajuato; ${ }^{5}$ Delegación del Instituto Mexicano del Seguro Social (IMSS), León, Guanajuato; ${ }^{6}$ Departamento de Patología Clínica y Molecuar, Instituto NIDIAC, Durango, Durango. México

${ }^{\dagger}$ Autor fallecido el 26 de agosto del 2020.

\section{Resumen}

Objetivo: Describir el perfil de multirresistencia (MDR), resistencia extendida (XDR) y panresistencia (PDR) a antibacterianos en aislados de muestras de pacientes de un hospital privado de tercer nivel en el norte de México. Método: Se realizó un estudio retrospectivo durante el periodo comprendido de febrero de 2016 a abril de 2019. A partir de 156 muestras clínicas de orina, heridas, sangre, expectoración y otros fluidos se aislaron 166 bacterias; 10 de las muestras incluyeron dos microorganismos. Los microrganismos aislados se clasificaron en MDR, XDR o PDR. Resultados: El 78\% de los aislados gramnegativos y el 69\% de los aislados grampositivos mostraron MDR, XDR o PDR. Staphylococcus epidermidis fue la bacteria grampositiva con multirresistencia más frecuentemente aislada. Escherichia coli y Klebsiella sp. se encontraron entre los gramnegativos MDR más frecuentes. En dos casos, los aislados clínicos de Pseudomonas aeruginosa procedentes de la unidad de cuidados intensivos neonatales mostraron PDR. Conclusión: Los servicios de terapia intensiva, cirugía y unidad de cuidados intensivos neonatales merecen especial atención por la alta proporción de aislados MDR y la presencia de PDR a causa de $P$. aeruginosa.

Palabras clave: Bacteria. Multirresistencia. Resistencia extendida. Panresistencia. México.

\section{ABSTRACT}

Objective: To describe the profile of multidrug-resistance (MDR), extensively resistance (XDR) and pandrug-resistance (PDR) to antibacterial drugs in isolates from patient samples from a third level private hospital in the North of Mexico. Method: $A$ retrospective study was carried out during the period from February 2016 to April 2019. From 156 clinical samples of urine, wounds, blood, expectoration and other fluids, 166 bacteria were isolated; 10 samples included two microorganisms. Isolated microorganisms were classified into MDR, XDR or PDR. Results: $78 \%$ of the Gram negative and $69 \%$ of the Gram positive isolates showed MDR, XDR or PDR. Staphylococcus epidermidis was the most frequently isolated MDR Gram positive bacteria. Escherichia coli and Klebsiella sp. were among the most frequent MDR Gram negative. In two cases, the clinical isolates of Pseudomonas aeruginosa from the neonatal intensive care unit showed PDR. Conclusions: The intensive care, surgery

\section{Correspondencia:}

*Cecilia Ishida-Gutiérrez

Circuito Universitario 31109

Campus UACH II

Fecha de recepción: 09-04-2020

Fecha de aceptación: 25-05-2020

C.P. 31125, Chihuahua, Chih., México

E-mail: ishida.cecilia@gmail.com 
and neonatal intensive care unit services deserve special attention due to the high proportion of MDR isolates and the presence of $P D R$ due to $P$. aeruginosa.

Key words: Bacteria. Multidrug resistance. Extended resistance. Pan-resistance. Mexico.

\section{Introducción}

La resistencia a antimicrobianos se refiere a los procesos que presentan los microorganismos (bacterias, virus, hongos y parásitos) que inciden y hacen ineficaces a los medicamentos utilizados en su tratamiento'.

La escasez, y en algunos casos ausencia, de medicamentos eficaces para el tratamiento de enfermedades infecciosas tiene consecuencias graves. Los datos de la Organización Mundial de la Salud (OMS) indican que la resistencia bacteriana ocasionó 700,000 muertes en 2015; esta cifra, según el estudio realizado por O'Neill², podría ascender a 10 millones en 2050 si no se llevan a cabo intervenciones para revertir la tendencia. Según proyecciones recientes de la OMS, en los siguientes 30 años las muertes por bacterias resistentes podrían ser más frecuentes que las atribuidas al cáncer ${ }^{3,4}$. En terminología económica, la resistencia es una externalidad negativa, es decir, tiene un efecto indeseable en otras personas además de la inmediata en el consumidor del antibiótico ${ }^{5}$.

Un creciente número de infecciones, como neumonía, tuberculosis, septicemia y gonorrea, son cada vez más difíciles y a veces imposibles de tratar, a medida que los antimicrobianos van perdiendo efica$\mathrm{cia}^{1,6}$. Nunca la amenaza de resistencia a los antimicrobianos ha sido más inmediata y la necesidad de soluciones más urgente, dice el Dr. Tedros Adhanom Ghebreyesus, Director General de la OMS.

Si bien los informes de agentes antibacterianos en desarrollo son innovadores, pasarán años antes de que lleguen a los pacientes y los nuevos tratamientos por sí solos no serán suficientes para combatir la amenaza de la resistencia a los antimicrobianos; por tal motivo, la OMS trabaja con diferentes países para mejorar la prevención y el control de las infecciones, y para fomentar el uso apropiado de los antibióticos existentes y futuros ${ }^{7,8}$.

La resistencia a los antimicrobianos puede ser natural (intrínseca) o adquirida. La resistencia natural es resultado de propiedades específicas del microorganismo y su aparición es previa al uso de los antibióticos. La resistencia adquirida se define como la pérdida de la sensibilidad a las concentraciones terapéuticas utilizadas de un medicamento particular.
En sentido estricto, se considera multirresistente (MDR, multidrug-resistant) al microorganismo que presenta resistencia adquirida in vitro a más de un fármaco antibacteriano, pero esta MDR puede estratificarse en niveles. Las definiciones internacionales de MDR, resistencia extendida (XDR, extensively drug-resistant) y panresistencia (PDR, pandrug-resistant) fueron propuestas en $2012^{9}$. Se considera MDR per se al microorganismo no sensible al menos a un antimicrobiano de tres o más familias farmacológicas. La XDR la presenta el microorganismo no sensible a un fármaco de todas las categorías, menos dos o una categorías. La PDR se refiere al microorganismo resistente a todos los fármacos de todas las categorías. El uso adecuado de estas definiciones permite una mejor comprensión de la extensión del problema de la resistencia y hace posible la comparación de los datos de vigilancia epidemiológica entre instituciones, regiones y países ${ }^{9}$. Para lograr diseñar y llevar a cabo intervenciones dirigidas cuyos resultados puedan ser medidos, es imprescindible que la información acerca de la resistencia a los antimicrobianos se organice en un conocimiento específico de las características y la magnitud del problema. El presente estudio tiene como objetivo conocer el perfil de resistencia bacteriana en un hospital privado de tercer nivel.

\section{Método}

Se realizó un estudio observacional, transversal y retrospectivo durante el periodo comprendido de febrero de 2016 a abril de 2019 en un hospital privado de tercer nivel de atención, ubicado en la ciudad de Chihuahua, Chihuahua, México. Se calculó el tamaño de la muestra de acuerdo con el número de muestras clínicas con cultivo existentes durante el mencionado periodo. Se aislaron 166 bacterias (Tabla 1). La identificación de los microorganismos se efectúo a partir de diferentes cultivos y su sensibilidad a los antibióticos se determinó con el sistema automatizado BD Phoenix cumpliendo con las políticas internas del laboratorio de la institución y los estándares del Clinical and Laboratory Standards Institute $(\mathrm{CLSI})^{10}$. Los microrganismos aislados se clasificaron en MDR, XDR o PDR de 
Tabla 1. Tipo de muestras clínicas, número de aislados bacterianos y nivel de resistencia

\begin{tabular}{|c|c|c|c|c|c|c|}
\hline \multirow[t]{2}{*}{ Muestra clínica } & \multirow[t]{2}{*}{ N. ${ }^{0}$ de muestras } & \multirow[t]{2}{*}{ N. ${ }^{\circ}$ de aislados } & \multicolumn{4}{|c|}{ Nivel de resistencia } \\
\hline & & & MDR & XDR & PDR & No MDR \\
\hline Orina & 23 & 25 & 16 & 0 & 0 & 9 \\
\hline Exudado de herida/escara & 21 & 24 & 17 & 1 & 0 & 6 \\
\hline Hemocultivo/catéter & 48 & 48 & 31 & 4 & 1 & 12 \\
\hline Expectoración/secreción bronquial & 48 & 53 & 40 & 2 & 1 & 10 \\
\hline Otras & 16 & 16 & 10 & 0 & 0 & 6 \\
\hline Total & 156 & 166 & 114 & 7 & 2 & 43 \\
\hline
\end{tabular}

MDR: multirresistencia; PDR: panresistencia; XDR: resistencia extendida.

Tabla 2. Clasificación de las bacterias aisladas según la tinción de Gram y especies más frecuentes

\begin{tabular}{lll}
\hline Clasificación & $\mathbf{n}(\%)$ & $\begin{array}{l}\text { Principales representantes } \\
\text { (en orden descendente) }\end{array}$ \\
\hline Gramnegativas & $96(58)$ & $\begin{array}{l}\text { E. coli, P. aeruginosa, } \\
\text { K. pneumoniae }\end{array}$ \\
Grampositivas & $70(42)$ & $\begin{array}{l}\text { S. epidermidis, E. faecalis, } \\
\text { S. aureus }\end{array}$ \\
Total & $166(100)$ & \\
\hline
\end{tabular}

acuerdo con las definiciones del Centro Europeo para la Prevención y Control de Enfermedades (ECDC) y los Centers for Disease Control and Prevention (CDC) de los Estados Unidos de Norteamérica-11. Se extrajo información adicional de las historias clínicas, como el servicio de ingreso. Las variables de interés se registraron en una base de datos.

Se contó con la aprobación del Comité de Investigación de la Facultad de Medicina y Ciencias Biomédicas (Cl-057-19), y no se utilizaron datos personales de pacientes. Las pruebas estadísticas empleadas fueron de tipo descriptivo. Se obtuvieron valores porcentuales por especie de bacterias y área hospitalaria.

\section{Resultados}

Se cultivaron 156 muestras clínicas de diversos fluidos biológicos: orina $(n=23)$, exudado de heridas 0 escaras $(n=21)$, sangre y punta de catéter $(n=48)$, expectoración y secreción bronquial $(n=48)$, y otros (p. ej., líquido cefalorraquídeo, peritoneal y pulmonar; $n=16$ ). A partir de estas muestras se caracterizaron 166 cepas bacterianas debido a que en 10 de las muestras clínicas analizadas se aislaron dos bacterias (Tabla 1).

El 58\% (96/166) de las bacterias fueron gramnegativas, encontrándose entre las más frecuentes Escherichia coli (29/166), Pseudomonas aeruginosa (18/166) y Klebsiella pneumoniae (14/166); entre las bacterias grampositivas (70/166) más comunes se encontraron: Staphylococcus epidermidis (22/166), Enterococcus faecalis (14/166) y Staphylococcus aureus (10/166) (Tabla 2).

Se empleó el perfil de sensibilidad a antibióticos para clasificar las bacterias como no MDR (cuando no presentaron multirresistencia), MDR, XDR o PDR. La figura 1 muestra que 75 bacterias gramnegativas (78\%) presentaron algún nivel de multirresistencia: se encontraron 66 con MDR, siete con XDR y, notoriamente, dos con PDR. Por su parte, 48 (69\%) aislados de bacterias grampositivas resultaron ser MDR. La caracterización por especie y su nivel de MDR se muestran en la tabla 3. Las 166 bacterias pertenecieron a una de 33 especies distintas; solo tres de las 33 especies aisladas no presentaron ningún nivel de MDR (Pseudomonas oryzihabitans, Citrobacter freundii y Staphylococcus schleiferi). P. aeruginosa mostró los tres niveles de resistencia múltiple (MDR: ocho aislados; XDR: tres aislados; PDR: dos aislados). Al menos un aislado de K. pneumoniae, Klebsiella aerogenes, Acinetobacter baumannii y complejo Burkholderia cepacia presentó MDR y XDR. Por último, 25 especies mostraron al menos un aislado con MDR. Esto nos demuestra que la multirresistencia en la muestra abarca todos los niveles y casi todas las especies incluidas.

En cuanto al tipo de muestra biológica y el nivel de resistencia (Tabla 1), todos los aislados procedentes de orina que presentaron multirresistencia pertenecieron al 


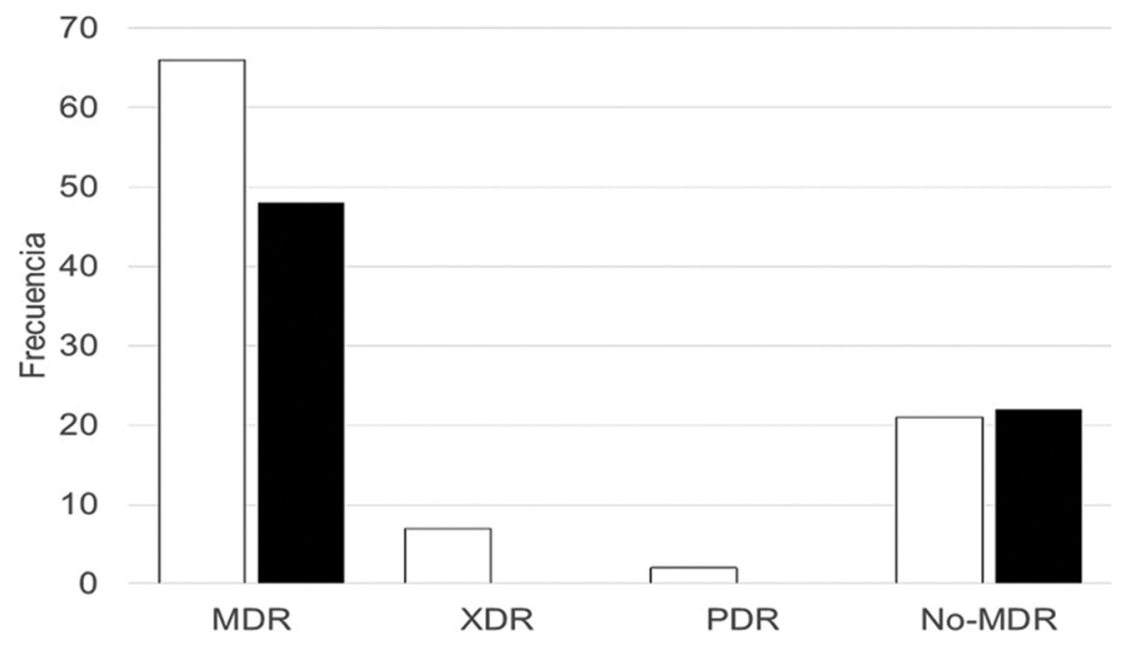

Figura 1. Distribución de aislados por nivel de multirresistencia y tinción. En blanco, aislados gramnegativos; en negro, aislados grampositivos. MDR: multirresistentes; PDR: panresistentes; XDR: con resistencia extendida.

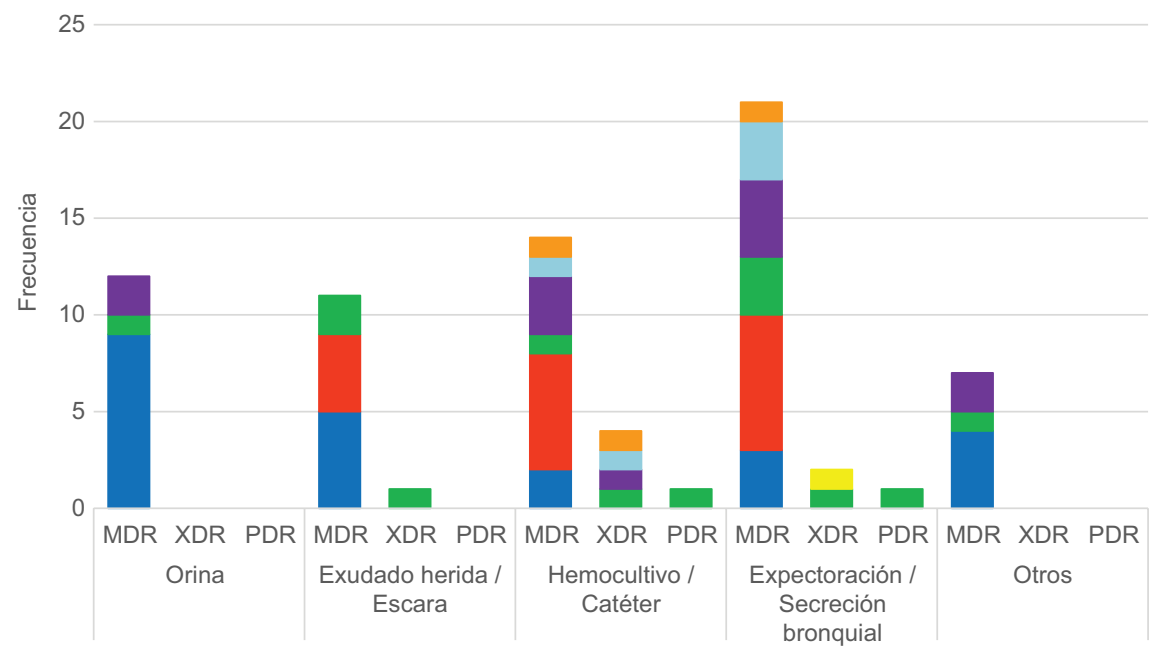

Figura 2. Microorganismos resistentes más frecuentes y distribución según el origen de la muestra: Escherichia coli (azul oscuro), Klebsiella pneumoniae (violeta), Pseudomonas aeruginosa (verde), Staphylococcus epidermidis (rojo), Acinetobacter baumannii (naranja), Klebsiella aerogenes (azul claro) y complejo Burkholderia cepacia (amarillo). MDR: multirresistentes; PDR: panresistentes; XDR: con resistencia extendida.

nivel MDR, es decir, no hubo aislados de orina con XDR ni PDR. En las muestras de exudado de herida o escara, por cada 17 aislados MDR se aisló uno con XDR. En las muestras de hemocultivo o catéter, por cada siete aislados MDR se aisló alrededor de uno con XDR. En las muestras de expectoración o secreción bronquial, por cada 20 aislados MDR se encontró uno con XDR. Los dos aislados de $P$. aeruginosa con PDR se obtuvieron de muestras de hemocultivo o catéter y de expectoración o secreción bronquial.

En la figura 2 se muestran las cinco especies más frecuentes en cada nivel de multirresistencia. En orina, como ya se ha mencionado, todos los aislados multirresistentes presentaron MDR y todos correspondieron a E. coli. En las muestras tomadas de exudado de herida o escara, las cepas multirresistentes más frecuentemente aisladas con nivel MDR fueron E. coli (cinco aislados) y $S$. epidermidis (cuatro aislados); hubo un solo aislado con XDR, que correspondió a $P$. aeruginosa, y no hubo aislados PDR. Los aislados obtenidos de hemocultivo o catéter fueron los que mostraron más variedad; $E$. coli siguió estando entre las bacterias más frecuentes con MDR (dos aislados), pero S. epidermidis se aisló con mayor frecuencia (6 aislados), y entre los aislados con MDR que les siguieron en frecuencia se encontraron $P$. aeruginosa (un 
Cirugía y Cirujanos. 2021;89(4)

Tabla 3. Distribución de los aislados por especie y nivel de resistencia

\begin{tabular}{|c|c|c|c|c|c|c|}
\hline \multirow[t]{2}{*}{ Especie } & \multirow[t]{2}{*}{ Tinción de Gram } & \multirow[t]{2}{*}{ Frecuencia } & \multicolumn{4}{|c|}{ Nivel de resistencia } \\
\hline & & & MDR & XDR & PDR & No MDR \\
\hline Escherichia coli & - & 29 & 23 & 0 & 0 & 6 \\
\hline Staphylococcus epidermidis & + & 22 & 17 & 0 & 0 & 5 \\
\hline Pseudomonas aeruginosa & - & 18 & 8 & 3 & 2 & 5 \\
\hline Enterococcus faecalis & + & 14 & 7 & 0 & 0 & 7 \\
\hline Klebsiella pneumoniae & - & 14 & 11 & 1 & 0 & 2 \\
\hline Staphylococcus aureus & + & 10 & 7 & 0 & 0 & 3 \\
\hline Staphylococcus hominis & + & 9 & 5 & 0 & 0 & 4 \\
\hline Staphylococcus haemolyticus & + & 7 & 6 & 0 & 0 & 1 \\
\hline Klebsiella aerogenes & - & 5 & 4 & 1 & 0 & 0 \\
\hline Enterobacter cloacae & - & 4 & 2 & 0 & 0 & 2 \\
\hline Acinetobacter baumannii & - & 4 & 2 & 1 & 0 & 1 \\
\hline Klebsiella oxytoca & - & 3 & 2 & 0 & 0 & 1 \\
\hline Stenotrophomonas maltophilia & - & 3 & 3 & 0 & 0 & 0 \\
\hline Proteus mirabilis & - & 2 & 1 & 0 & 0 & 1 \\
\hline Bacillus cereus & + & 2 & 2 & 0 & 0 & 0 \\
\hline Pseudomonas putida & - & 2 & 1 & 0 & 0 & 1 \\
\hline Streptococcus agalactiae & + & 2 & 1 & 0 & 0 & 1 \\
\hline Serratia liquefaciens & - & 1 & 1 & 0 & 0 & 0 \\
\hline Alcaligenes faecalis & - & 1 & 1 & 0 & 0 & 0 \\
\hline Enterobacter aerogenes & - & 1 & 1 & 0 & 0 & 0 \\
\hline Serratia marcescens & - & 1 & 1 & 0 & 0 & 0 \\
\hline Staphylococcus lentus & + & 1 & 1 & 0 & 0 & 0 \\
\hline Staphylococcus cohnii & + & 1 & 1 & 0 & 0 & 0 \\
\hline Pseudomonas oryzihabitans & - & 1 & 0 & 0 & 0 & 1 \\
\hline Citrobacter freundii & - & 1 & 0 & 0 & 0 & 1 \\
\hline Staphylococcus schleiferi & + & 1 & 0 & 0 & 0 & 1 \\
\hline Providencia rettgeri & - & 1 & 1 & 0 & 0 & 0 \\
\hline Achromobacter spp. & - & 1 & 1 & 0 & 0 & 0 \\
\hline Enterococcus faecium & + & 1 & 1 & 0 & 0 & 0 \\
\hline Salmonella spp. & - & 1 & 1 & 0 & 0 & 0 \\
\hline Complejo Burkholderia cepacia & - & 1 & 0 & 1 & 0 & 0 \\
\hline Pantoea agglomerans & - & 1 & 1 & 0 & 0 & 0 \\
\hline Sphingobacterium multivorum & - & 1 & 1 & 0 & 0 & 0 \\
\hline
\end{tabular}

aislado), K. pneumoniae (un aislado), K. aerogenes (un aislado) y Acinetobacter baumannii (un aislado). Por último, como se mencionó anteriormente, uno de los dos aislados PDR de $P$. aeruginosa provino de una muestra de hemocultivo o catéter y el otro de expectoración o secreción bronquial. En este último fluido, el perfil de bacterias semejó en composición al de hemocultivo o catéter; se encontraron siete aislados de $S$. 
Tabla 4. Distribución de aislados por servicio de ingreso y nivel de resistencia

\begin{tabular}{lcccccc}
\hline Servicio & $\begin{array}{c}\mathbf{N} .{ }^{\circ} \text { de } \\
\text { aislados }\end{array}$ & $\begin{array}{c}\text { Aislados MDR } \\
\mathbf{n}(\%)\end{array}$ & $\begin{array}{c}\text { Aislados XDR } \\
\mathbf{n}(\%)\end{array}$ & $\begin{array}{c}\text { Aislados PDR } \\
\mathbf{n}(\%)\end{array}$ & $\begin{array}{c}\text { Total MDR+XDR+PDR } \\
\mathbf{n}(\%)\end{array}$ & $\begin{array}{c}\text { Total no MDR } \\
\mathbf{n}(\%)\end{array}$ \\
\hline Cirugía & 23 & $17(74)$ & 0 & 0 & $17(74)$ & $6(26)$ \\
Medicina interna & 30 & $16(53)$ & 0 & 0 & $16(53)$ & $14(47)$ \\
Ginecología y obstetricia & 5 & $4(80)$ & 0 & 0 & $4(80)$ & $1(20)$ \\
Pediatría & 5 & $2(40)$ & 0 & 0 & $2(40)$ & $3(60)$ \\
$\begin{array}{l}\text { Unidad de cuidados intensivos } \\
\text { neonatales }\end{array}$ & 31 & $19(61)$ & $3(10)$ & & $2(6)$ & $7(23)$ \\
Unidad de terapia intensiva & 72 & $56(78)$ & $4(5)$ & 0 & $60(83)$ & $12(17)$ \\
\hline
\end{tabular}

MDR: multirresistentes; PDR: panresistentes; XDR: con resistencia extendida.

epidermidis, cuatro aislados de K. pneumoniae y tres aislados cada uno de E. coli, $P$. aeruginosa y $K$. aerogenes. Entre los aislados con XDR de expectoración o secreción bronquial más frecuentes se identificaron $P$. aeruginosa y miembros del complejo $B$. cepacia. Como material suplementario, en el Anexo 1 se detallan todos microorganismos aislados por especie, la muestra de donde proceden y el nivel de resistencia (MDR, XDR o PDR).

Con el propósito de explorar la magnitud del problema de la multirresistencia en las distintas secciones del hospital, los aislados se clasificaron de acuerdo con el servicio y el nivel de multirresistencia (Tabla 4). Se encontró un $53 \%$ a $83 \%$ de aislados con algún nivel de multirresistencia en los diferentes servicios, siendo la unidad de terapia intensiva (83.3\%), el servicio de ginecología y obstetricia $(80 \%)$, y el servicio de cirugía $(73.9 \%)$ los más afectados en cuanto a proporción de aislados con multirresistencia. La unidad de cuidados intensivos neonatales presentó aislados bacterianos con los tres niveles de multirresistencia; de este servicio se aislaron las dos cepas de $P$. aeruginosa con PDR (Tablas 3-4). No hubo aislados bacterianos procedentes del servicio de urgencias.

\section{Discusión}

La resistencia bacteriana es un problema altamente significativo de trascendencia mundial que amerita su vigilancia y control continuo para limitarla. La OMS ha implementado estrategias ${ }^{12,13}$ que son primordiales para direccionar las acciones. Los resultados de este estudio muestran que, así como ocurre en todo el mundo ${ }^{14-16}$, la multirresistencia en los aislados de nuestro hospital afecta tanto a grampositivos como a gramnegativos (Fig. 1). Como en otros estudios ${ }^{17}$, E. coli y Klebsiella sp. se encontraron entre los gramnegativos multirresistentes más frecuentes, y Pseudomonas ocupó el segundo lugar en cuanto a frecuencia entre los multirresistentes gramnegativos, y en extensión de multirresistencia ocupó el primer lugar, ya que dos aislados clínicos presentaron PDR. En cuanto a los microorganismos grampositivos, S. epidermidis fue la bacteria con multirresistencia más frecuentemente aislada, a diferencia de otros estudios ${ }^{17}$ en los que $S$. aureus suele ocupar el primer lugar; 17 de los 22 aislados clínicos de $S$. epidermidis en esta muestra presentaron MDR, lo cual es de considerar dado su potencial para participar en infecciones nosocomiales y formación de biopelículas. E. faecalis también se aisló de manera frecuente y el $50 \%$ de estos aislados presentaron MDR.

Es alarmante observar que únicamente uno de cada cuatro aislados clínicos de esta muestra no presenta multirresistencia (44 de 166 aislados) (Fig. 1), y que de las 33 especies identificadas solo tres no presentaron ningún nivel de multirresistencia (Tabla 3). Este hecho señala una resistencia generalizada y confirma la gravedad del problema en la región norte del país ${ }^{17}$.

El análisis de la multirresistencia según el fluido señala que el problema es de mayor complejidad y gravedad en los pacientes con infecciones en la sangre (o al menos en un catéter) o en las vías respiratorias, ya que en estos fluidos fue donde se encontró el mayor porcentaje de aislados multirresistentes de cada nivel (MDR, XDR y PDR), la mayor variedad de bacterias y los dos aislados PDR.

En cuanto a los servicios hospitalarios, los de terapia intensiva, ginecología y obstetricia, y cirugía requieren atención por la alta proporción de aislados 
bacterianos con multirresistencia en muestras clínicas provenientes de pacientes de dichos servicios; además, el servicio de cuidados intensivos neonatales requiere atención especial por ser fuente de aislados PDR.

La información obtenida permitirá implementar medidas dirigidas a los servicios con mayores problemas de multirresistencia y enfocar el uso adecuado de los antibióticos en este hospital para el tratamiento de las enfermedades infecciosas ocasionadas por los microorganismos que se encontraron con mayor multirresistencia, tanto en frecuencia como en gravedad. El estudio de otros factores posiblemente involucrados se encuentra en proceso.

\section{Conclusiones}

En nuestro estudio, la multirresistencia a los antibacterianos abarca hasta el $75 \%$ de los aislados clínicos y alcanza una extensión de PDR en el 1.2\% de los casos. Los aislados multirresistentes más frecuentes fueron E. coli, $P$. aeruginosa, $S$. epidermidis y K. pneumoniae; estas especies y los servicios de cuidados intensivos, cirugía, ginecología y obstetricia, y la unidad de cuidados intensivos de neonatos son áreas de prioridad para el estudio de factores de riesgo y la implementación de intervenciones.

\section{Agradecimientos}

Al personal del laboratorio de la institución participante, por el apoyo brindado. LACS recibe la beca CONACYT con número de apoyo 763423.

\section{Responsabilidades éticas}

Protección de personas y animales. Los autores declaran que para esta investigación no se han realizado experimentos en seres humanos ni en animales.

Confidencialidad de los datos. Los autores declaran que en este artículo no aparecen datos de pacientes.

Derecho a la privacidad y consentimiento informado. Los autores declaran que en este artículo no aparecen datos de pacientes.

\section{Conflicto de intereses}

Los autores declaran que no existen conflictos de intereses en la realización de este trabajo.

\section{Anexo}

Puede consultarse material adicional en el Anexo disponible en: https://doi.org/10.24875/CIRU.20000304.

\section{Bibliografía}

1. Valdes M. La resistencia microbiana en el contexto actual y la importancia del conocimiento y aplicación en la política antimicrobiana. Revhabanera.sld.cu. 2019. (Consultado el 10 de junio de 2019)]. Disponible en: http://www.revhabanera.sld.cu/index.php/rhab/article/view/2013

2. O'Neill J. Review on Antimicrobial Resistance Antimicrobial Resistance: Tackling a crisis for the health and wealth of nations. London: Review on Antimicrobial Resistance. 2014. Disponible en: https://amr-review.org/sites/ default/files/AMR\%20Review\%20Paper\%20-\%20Tackling\%20a\%20crisis $\% 20$ for\%20the\%20health\%20and\%20wealth\%20 of\%20nations_1.pdf.

3. Willyard C. The drug-resistant bacteria that pose the greatest health threats. Nature. 2017;543:15.

4. Osman M, Al Mir H, Rafei R, Dabboussi F, Madec J, Haenni M, et al. Epidemiology of antimicrobial resistance in Lebanese extra-hospital settings: an overview. J Glob Antimicrob Resist. 2019;17:123-9.

5. Ahmad M, Khan A. Global economic impact of antibiotic resistance: a review. J Glob Antimicrob Resist. 2019;19:313-6.

6. Ventola C. The antibiotic resistance crisis. Part 1: causes and threats. PubMed Central (PMC). 2015. (Consultado el 10 de junio de 2019)]. Disponible en: https://www.ncbi.nlm.nih.gov/pmc/articles/PMC4378521/

7. World Health Organization. Antibacterial agents in clinical development: an analysis of the antibacterial clinical development pipeline. Geneva: WHO; 2019. Licence: CC BY-NC-SA 3.0 IGO.

8. World Health Organization. Antibacterial agents in preclinical development: an open access database. Geneva: WHO; 2019. WHO/EMP/ IAU/2019.12. Licence: CC BY-NC-SA 3.0 IGO.

9. Magiorakos A, Srinivasan A, Carey R, Carmeli Y, Falagas M, Giske C, et al. Multidrug-resistant, extensively drug-resistant and pandrug-resistant bacteria: an international expert proposal for interim standard definitions for acquired resistance. Clin Microbiol Infect. 2012;18:268-81.

10. Clinical and Laboratory Standards Institute. Performance Standards for Antimicrobial Susceptibility Testing. 26th ed. CLSI supplement M100S. Wayne, PA: CLSI; 2016.

11. The European Committee on Antimicrobial Susceptibility Testing. Breakpoint tables for interpretation of MICs and zone diameters. Version 10.0, 2020. Disponible en: http://www.eucast.org.

12. World Health Organization. The Global Strategy and Plan of Action on Public Health, Innovation and Intellectual Property (GSPOA). (Consultado el 17 de junio de 2019). Disponible en: http://who.int/phi/implementation/phi globstat_action/en

13. Organización Mundial de la Salud. Boletín de la OMS. 1983. 17. 61. p. 423-33. (Consultado el 17 de junio de 2019.) Disponible en: http://www. who.int/bulletin/es/

14. Lozano C, Torres C. Actualización en la resistencia antibiótica en Gram positivos. Enferm Infecc Microbiol Clin. 2017;35:2-8.

15. Fariñas M, Martínez-Martínez L. Infecciones causadas por bacterias gramnegativas multirresistentes: enterobacterias, Pseudomonas aeruginosa, Acinetobacter baumannii y otros bacilos gramnegativos no fermentadores. Enferm Infecc Microbiol Clin. 2013;31:402-9.

16. Lutgring J. Carbapenem-resistant Enterobacteriaceae: an emerging bacterial threat. Semin Diagn Pathol. 2019;36:182-6.

17. Garza-González E, Morfín-Otero R, Mendoza-Olazarán S, Bocanegra-lbarias $\mathrm{P}$, Flores-Treviño S, Rodríguez-Noriega $\mathrm{E}$, et al. A snapshot of antimicrobial resistance in Mexico. Results from 47 centers from 20 states during a six-month period. PLoS One. 2019;14:e0209865. 


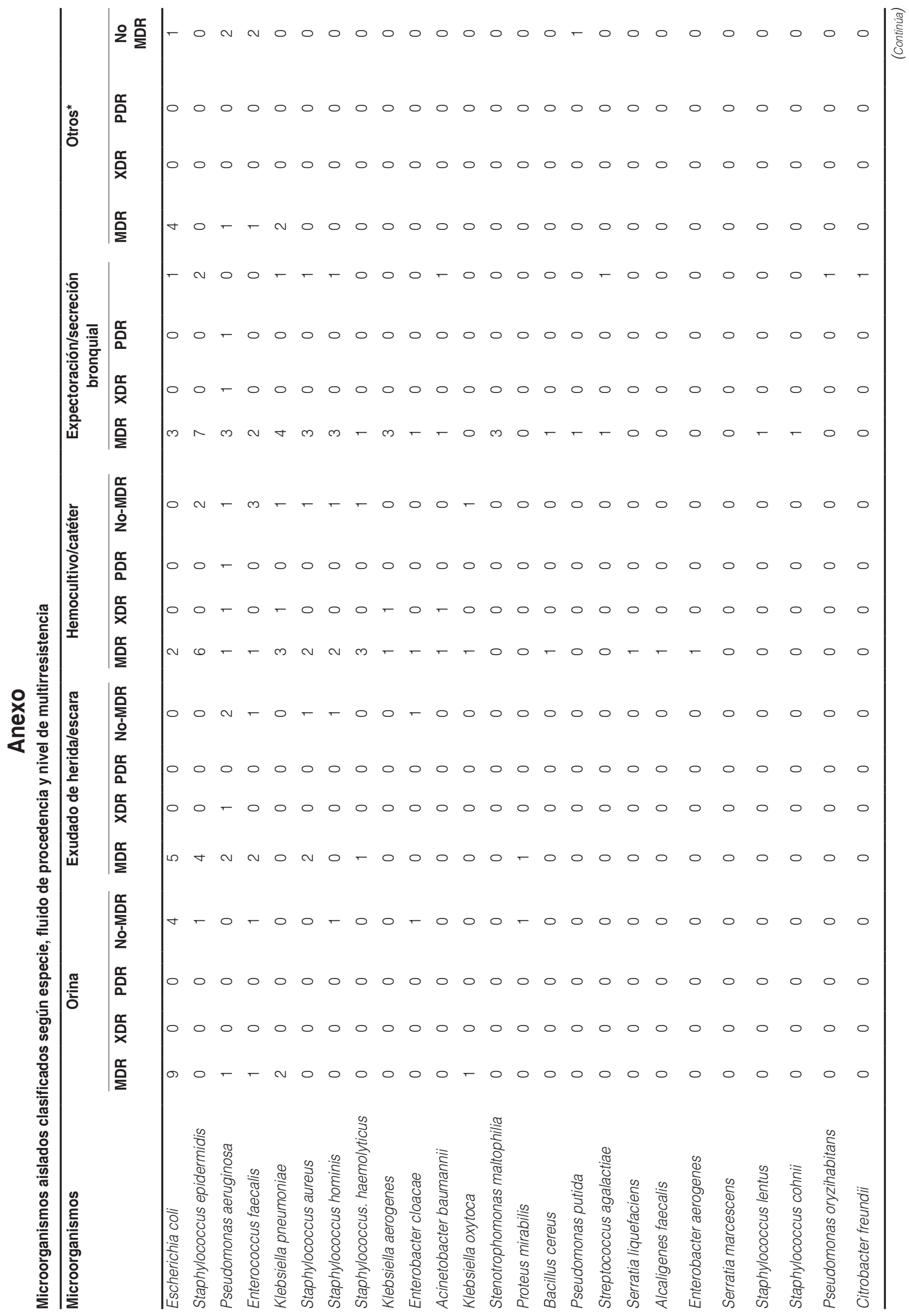




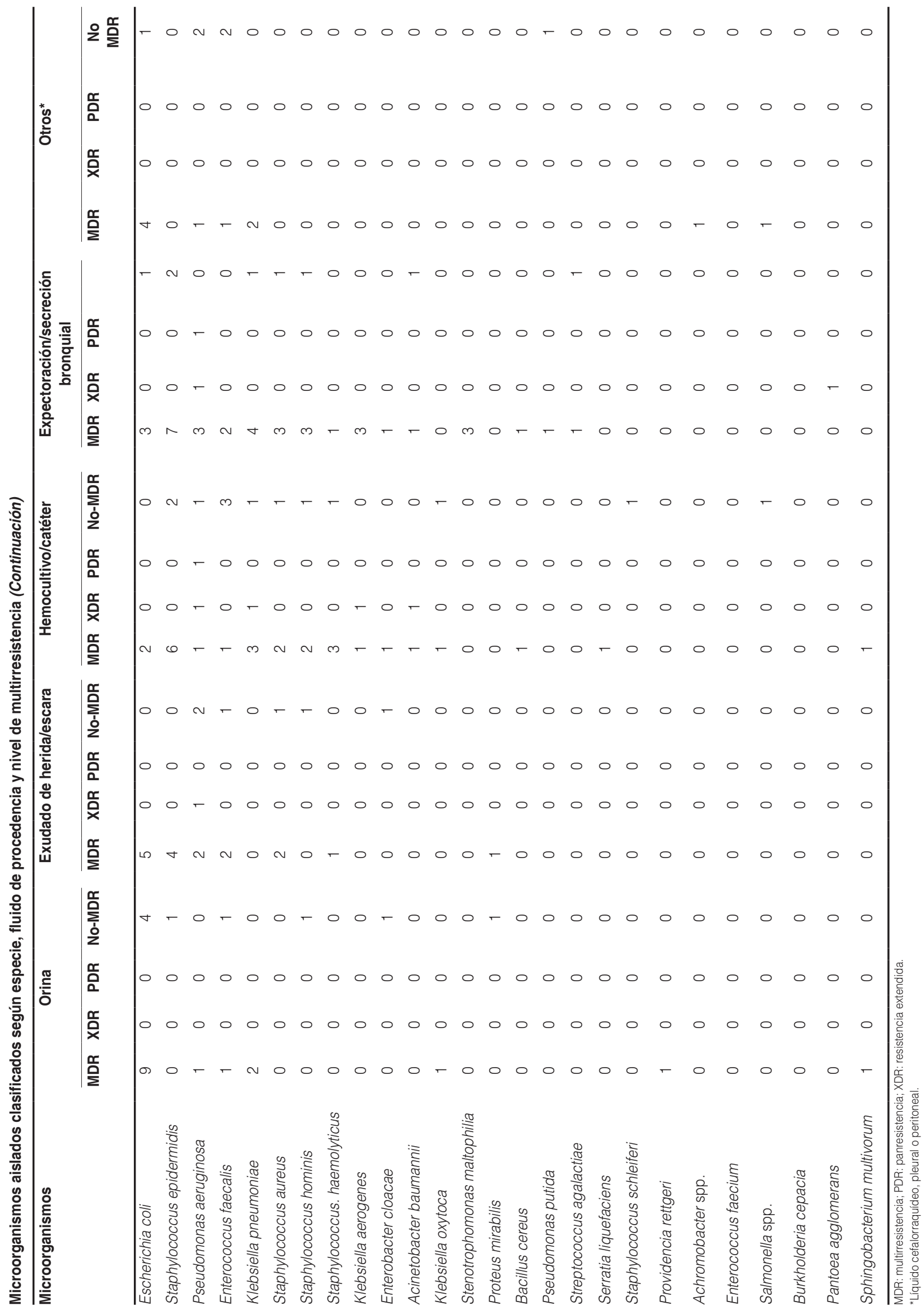

